\title{
Challenges of Superdense Coding with Accelerated Fermions
}

\author{
Mehrnoosh Farahmand", Hosein Mohammadzadeh \\ Department of Physics, University of Mohaghegh Ardabili, Iran
}

Copyright $(2017$ by authors, all rights reserved. Authors agree that this article remains permanently open access under the terms of the Creative Commons Attribution License 4.0 International License

\begin{abstract}
Two particles, even being far from each other have quantum correlation as a result of the existence of entanglement between them. Therefore, information can be shared by entangled particles, sitting in separate places. Superdense coding is one of the quantum protocols that rely on entanglement. In this paper, we review superdense coding with a non-inertial observer in the beyond single mode approximation and investigate the probability of success for superdense coding. We analyze the mutual information due to the effects of acceleration on the quantum and classical correlations of the state. Entanglement behavior is studied considering an entanglement measure the so-called the concurrence. Comparing the mutual information and the concurrence with the probability of superdense coding is shown that quantities have different behaviors, particularly, when the beyond single mode approximation plays a powerful role.
\end{abstract}

Keywords Superdense Coding, Entanglement, Non-Inertial Frame

\section{Introduction}

Entanglement has central importance in quantum information theory. Our current understanding of the universe reveals that it can be best described by relativistic physics and so, many implementations of quantum information tasks require relativistic system. In this sense, relativistic effects in quantum information have been exposed in vast domain of researches.

It has been shown that entanglement in the limit of infinite acceleration is non-zero between spinor modes in single mode approximation [1]. Recently, entanglement treatments and its applications in quantum information processing have been investigated for non-inertial observers in the single mode approximation [2, 3]. In order to understand how entanglement plays a role in the presence of Rindler horizon, the results have been extended to the beyond single mode approximation $[4,5,6]$.

The superdense coding by a non-inertial particle has been studied in beyond single mode approximation. The main purpose of this research is to study how the quantum and classical correlations and particularly, the entanglement of the state can be useful for superdense coding by a non-inertial observer in beyond single mode approximation.

We compare the probability of success in superdense coding with the quantum and classical correlations, the mutual information and a common entanglement measure, the concurrence [7, 8]. It is shown these quantities have different behaviours in beyond single mode approximation.

\section{Superdense Coding}

Superdense coding process begins with a pair of entangled two-level particles that is shared between Alice, as sender, and Bob, as receiver [9, 10]. An EPR pair in the two-dimension Hilbert space of the two particles, i. e., a Bell state as a maximally entangled state is used [11]. Alice and Bob share a Bell state, $\left|\phi_{00}\right\rangle_{A, B}$, as follows

$$
\left|\phi_{00}\right\rangle_{A, B}=\frac{1}{\sqrt{2}}(|0\rangle|0\rangle+|1\rangle|1\rangle)
$$

where subscripts A and B show Alice's qubit and Bob's one, respectively. Alice wants to send a two-bit message, 00, 01, 10 , or 11 , to Bob. She operates one of the four unitary operators, $\left\{\mathrm{I}, \sigma_{z}, \sigma_{x}, i \sigma_{y}\right\}$, on her qubit. By this operation the initial Bell state, Eq. (1), transforms to one of the four orthonormal Bell states. Then she transmits her manipulated qubit to Bob. Bob performs a measurement on the Bell-basis, yielding one of the four distinctive results. Therefore, based on the outcome, the initial two-bit message is distinguishable. In deede, under the superdense coding process, a classical two-bit message is encoded by one of the four Bell states, by the sender, and the receiver decodes this quantum state by a suitable measurement and achieves the original information. 


\section{Superdense Coding in Non-Inertial Frame}

Alice and Bob, as two inertial observers, start the process by sharing a maximally entangled two-qubit state, an EPR pair, such as Eq. (1). Consider Alice remains at rest and Bob starts to accelerate uniformly. Now, he is named Rob. As is seen in Figure 1, Rob's trajectory in Minkowski coordinates is a hyperbola, indicating in terms of Rindler coordinates as follows.

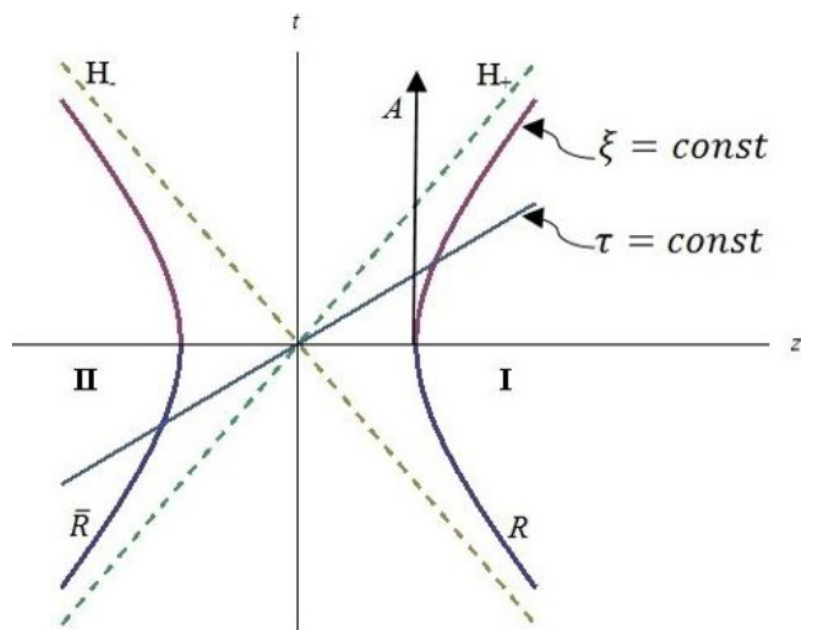

Figure 1. The probability of success in terms of the acceleration parameter, $r$, and the presence probability, $q_{r}$

$$
(z, t)= \pm\left(\frac{e^{a \xi}}{a} \cosh (a \tau), \frac{e^{a \xi}}{a} \sinh (a \tau)\right)
$$

Where $\tau$ is the Rob's proper time, $a$ is an arbitrary reference acceleration and $\frac{e^{a \xi}}{a}$ is the proper acceleration of Rob. The horizons $H_{ \pm}$are obtained by lines of 45 degree, represent proper times $\tau= \pm \infty$ in the limit $\xi \rightarrow-\infty$. The right and left half of Mikowski planne are Rindler wedge $I$ and $I I$, such that Rob and the fictitious observer, anti-Rob, are constrained to move in these regions, respectively. No information can propagate between these regions because of causally disconnection.

The Minkowski vacuum and one particle modes from Rob's view point are expanded in terms of the corresponding Rindler vacuum and one particle modes in the regions $I$ and $I I[1,4]$, as follows.

$$
\begin{gathered}
|0\rangle_{R}=\cos r|0\rangle_{I}|0\rangle_{I I}+\sin r|1\rangle_{I}|1\rangle_{I I}, \\
|1\rangle_{R}=q_{l}|0\rangle_{I}|1\rangle_{I I}+q_{r}|1\rangle_{I}|0\rangle_{I I}
\end{gathered}
$$

where $r=\tan ^{-1}(\exp (-\pi \Omega))$ is the parameter equal to acceleration with $\Omega \equiv \omega /(a / c)$, which is the ratio of the frequency observed by observers, $\omega$, to the naturally occurring frequency in the problem, $\frac{a}{c} \cdot q_{r}$ and $q_{l}$, the presence probability of particle in right or left Rindler regions, respectively, are the complex numbers that satisfy $q_{r}^{2}+q_{1}^{2}=1$ and $q_{r} \in[0,1]$. For simplicity, we only choose the cases that $q_{r}$ and $q_{l}$ are real. Therefore, the initially shared state, Eq. (1) is rewritten in terms of left and right Rindler regions such as follows

$$
\left|\phi_{00}\right\rangle_{A, I, I I}=\frac{1}{\sqrt{2}}\left\{\cos r|000\rangle+\sin r|011\rangle+q_{l}|110\rangle+q_{r}|101\rangle\right\},
$$

where $|a b c\rangle=|a\rangle_{A}|b\rangle_{I}|c\rangle_{I I}$. Alice applies one of the operators, $\left\{\mathrm{I}, \sigma_{z}, \sigma_{x}, i \sigma_{y}\right\}$, to her qubit.

$$
\begin{aligned}
\mathrm{I}\left|\phi_{00}\right\rangle_{A, I, I I} & =\frac{1}{\sqrt{2}}\left\{\cos r|000\rangle+\sin r|011\rangle+q_{l}|101\rangle+q_{r}|110\rangle\right\}, \\
\sigma_{z}\left|\phi_{00}\right\rangle_{A, I, I I} & =\frac{1}{\sqrt{2}}\left\{\cos r|000\rangle-\sin r|011\rangle+q_{l}|101\rangle-q_{r}|110\rangle\right\}, \\
\sigma_{x}\left|\phi_{00}\right\rangle_{A, I, I I} & =\frac{1}{\sqrt{2}}\left\{\cos r|010\rangle+\sin r|001\rangle+q_{l}|111\rangle+q_{r}|100\rangle\right\}, \\
i \sigma_{y}\left|\phi_{00}\right\rangle_{A, I, I I} & =\frac{1}{\sqrt{2}}\left\{-\cos r|010\rangle+\sin r|001\rangle-q_{l}|111\rangle+q_{r}|100\rangle\right\} .
\end{aligned}
$$

Suppose Alice has been used the operator I. This operator does not change the state (3). The Alice's manipulated qubit is sent to Bob. He has both qubits in his position. The Rindler region $I$ and $I I$ are causally disconnected and no information propagates between them. Bob is constrained to move in the region $I$, thus the density matrix is obtained by tracing out the region $I I$, as follows

$$
\begin{aligned}
\rho_{00}^{A, I}=\operatorname{Tr}_{I I} \rho^{A . I, I I}= & \frac{1}{2}\left\{\cos ^{2} r|00\rangle\left\langle 00\left|+\sin ^{2} r\right| 10\right\rangle\left\langle 10\left|+q_{l}^{2}\right| 01\right\rangle\left\langle 01\left|+q_{r}^{2}\right| 11\right\rangle\langle 11|+\right. \\
& \left.+\left(q_{l} \sin r|10\rangle\left\langle 01\left|+q_{r} \cos r\right| 00\right\rangle\langle 11|+\text { h.c }\right)\right\}
\end{aligned}
$$


Bob performs a Bell-basis measurement to decode the classical information, and gets the following results

Table 1. The Results of measurement in Bell-basis by Bob

\begin{tabular}{|c|c|c|c|c|}
\hline & $\left\langle\phi_{00}\left|\rho_{00}^{A, I}\right| \phi_{00}\right\rangle$ & $\left\langle\phi_{01}\left|\rho_{00}^{A, I}\right| \phi_{01}\right\rangle$ & $\left\langle\phi_{10}\left|\rho_{00}^{A, I}\right| \phi_{10}\right\rangle$ & $\left\langle\phi_{11}\left|\rho_{00}^{A, I}\right| \phi_{11}\right\rangle$ \\
\hline $\begin{array}{c}\text { Probability of finding } \rho_{00}^{A, I} \text { in the } \\
\text { Bell states }\end{array}$ & $\frac{\left(q_{r}+\cos r\right)^{2}}{4}$ & $\frac{\left(q_{r}-\cos r\right)^{2}}{4}$ & $\frac{\left(q_{l}+\sin r\right)^{2}}{4}$ & $\frac{\left(q_{l}-\sin r\right)^{2}}{4}$ \\
\hline
\end{tabular}

Table 1 shows that the probability of success for superdense coding is $P=\frac{1}{4}\left(q_{r}+\cos r\right)^{2}$. Thus Bob's measurement on the density matrix after tracing out the region $I I$ depends on the acceleration parameter, $r$, and the presence probability of particle in the left or in the right Rindler regions. The single mode approximation is recovered by $q_{r}=1$. The probability of success for superdense coding as a function of the acceleration parameter, $r$, and the presence probability of particle in the right Rindler region, $q_{r}$ is represented in Figure. 2. This function is decreasing with respect to $r$ and increasing with respect to $q_{r}$.

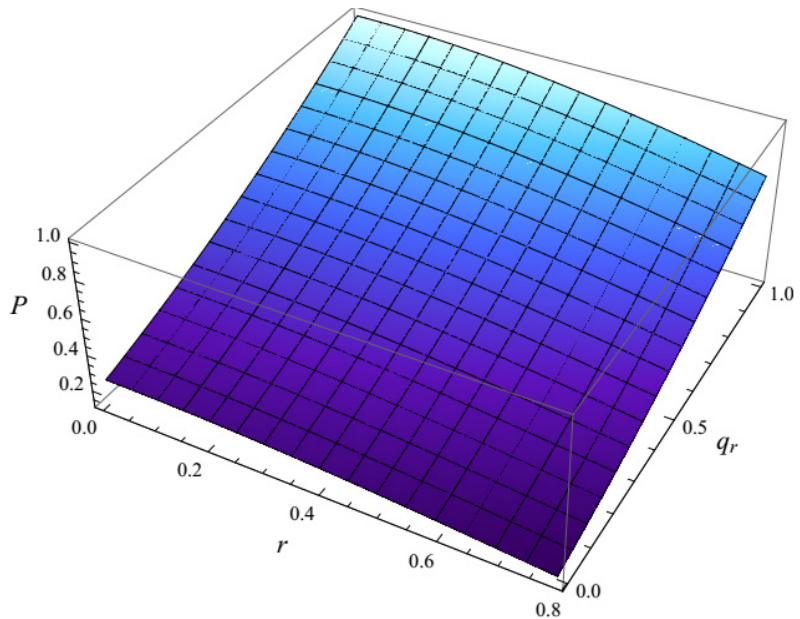

Figure 2. The probability of success in terms of the acceleration parameter, $r$, and the presence probability, $q_{r}$.

We calculate the mutual information to quantify the effects of acceleration on the quantum and classical correlations [7]. The mutual information is given by

$$
\mathfrak{T}\left(\rho^{A I}\right)=S\left(\rho^{A}\right)+S\left(\rho^{I}\right)-S\left(\rho^{A I}\right),
$$

where $S(\rho)=-\operatorname{Tr} \rho \log _{2} \rho$ denotes the von Neumann entropy of $\rho$, and $\rho^{A}$ and $\rho^{I}$ are reduced density matrices for subsystem $A$ and $I$, respectively. The mutual information can be calculated analytically. Since the corresponding expressions are quite long and not very enlightening, we give the plots of $\mathfrak{I}\left(\rho^{A I}\right)$ as a function of the acceleration, $r$, and the presence probability of particle in right Rindler region, $q_{r}$, in Figure 3.

When the acceleration is zero the correlations decrease with respect to the presence probability of particle in left Rindler region. In single mode approximation, $q_{r}=1$, the mutual information decreases with respect to the acceleration. It, from maximum value of 2 in absent of acceleration, approaches the value of 1 in the limit of infinite acceleration. Thus, the classical and quantum correlations become degraded with respect to the acceleration.

In the beyond single mode approximation, the situation is more complicated. The mutual information stays a decreasing function of the acceleration until the presence probability of particle in right Rindler region is more than the left one. But, it becomes an increasing function for more presence probability in left Rindler region. It is seem the correlations increase when the presence probability of particle in left Rindler region is more than the right one. However, in the limit of infinite acceleration, the mutual information approaches the value of 1 for all values of $q_{r}$.

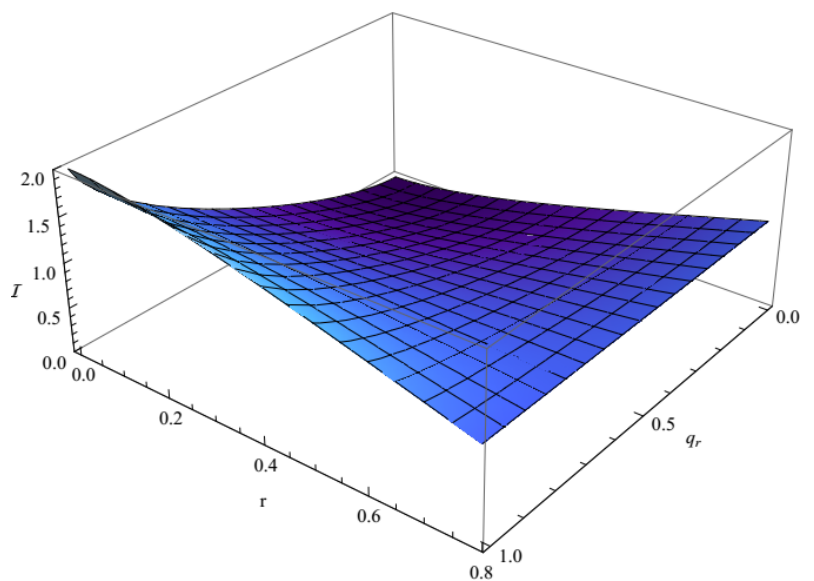

Figure 3. The mutual information in terms of the acceleration parameter, $r$, and the presence probability, $q_{r}$.

We employ the concurrence to provide further insight into the entanglement of the state $\rho_{00}^{A, I}$ in Eq. (5), [8]. The concurrence is defined by

$$
C\left(\rho_{A, I}\right):=\max \left\{0, \lambda_{1}-\lambda_{2}-\lambda_{3}-\lambda_{4}\right\},
$$

where $\lambda_{i} \geq \lambda_{i+1}, \lambda_{i}$ are the eigenvalues of the density matrix $\rho_{A, I}\left(\sigma_{y} \otimes \sigma_{y}\right) \rho_{A, I}{ }^{*}\left(\sigma_{y} \otimes \sigma_{y}\right), \sigma_{2}$ is the second Pauli matrix, and the asterisk is complex conjugation. Thus the concurrence for Eq. (5), is given by Figure 4.

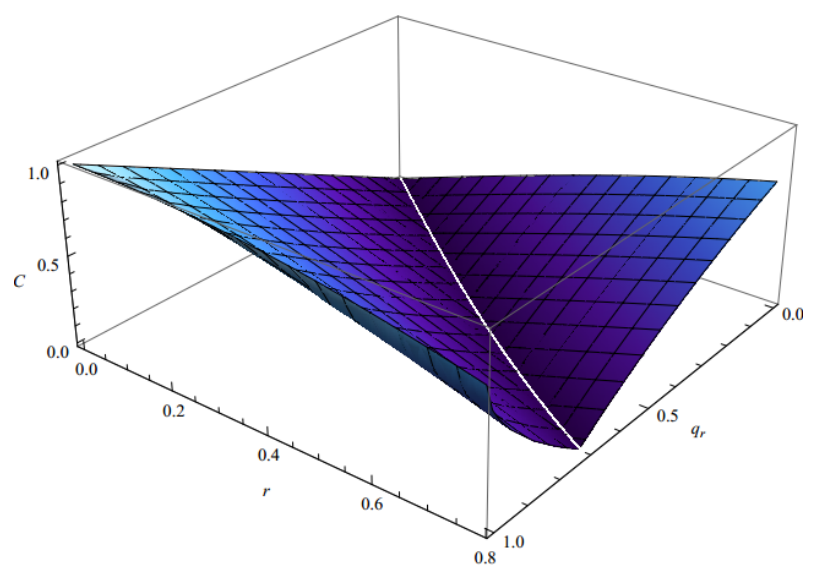

Figure 4. The concurrence in terms of the acceleration parameter, $r$, and the presence probability, $q_{r}$. 
Figure 4 shows the concurrence as a function of the acceleration parameter, $r$, and the presence probability of particle in the right Rindler region, $q_{r}$. In the single mode approximation, $q_{r}=1$, the concurrence is a descending function of acceleration, $r$.

The variations of entanglement in the interval $r=$ $[0, \pi / 4]$ is from 1 to $\frac{1}{\sqrt{2}}$. We saw the mutual information also decreases with respect to the acceleration. But, it varies from 2 to 1 in the interval $r=[0, \pi / 4]$. Thus the classical correlations also become degraded with increasing acceleration.

In beyond single mode approximation, so long as the particle is in the right Rindler region, i.e. it has a small probability to be in the left Rindler region, and for small values of acceleration, the concurrence has similar behaviours to the probability of success with respect to the acceleration and with respect to the presence probability of particle in the right Rindler region. It is clear that the concurrence is decreasing with increasing $r$ and with decreasing $q_{r}$. However, if the particle has larger probability of being in the left Rindler region and for large value of the acceleration, the concurrence has different behaviours with respect to the probability of success. The concurrence is an increasing function of $r$, and a descending function of $q_{r}$.

The analysis of the three above functions in the limit of infinite acceleration shows that with decreasing $q_{r}$, the probability of success is always a decreasing function, while the mutual information is a constant function with the value of 1 , and the concurrence first decreases and then becomes increased. Although, in this limit, the entanglement with respect to $q_{r}$ first show a decreasing behavior and then an increasing behavior, the total of quantum and classical correlations have no variation. However, the probability of success always stays a decreasing function of $q_{r}$.

Therefore, this correlation is not invariably suitable for superdense coding.

\section{Results and Discussion}

In the present work, we investigated the superdense coding by an accelerated observer in the beyond single mode approximation. We observed that the probability of success depends on the acceleration of references frames.

In Single mode approximation, $\mathrm{q}_{\mathrm{r}}=1$, and for $\mathrm{r}=0$, probability of success is maximum, i. e. $\mathrm{P}=1$, where is recovered the original superdense coding [9]. In this situation the mutual information and the concurrence are also maximum, i.e., $\mathfrak{T}=\mathbf{2}$ and $\mathrm{C}=1$. By increasing the acceleration, the probability of success, the mutual information and the concurrence are decreasing functions of the acceleration, as expected.

In the beyond single mode approximation, by increasing the acceleration, the probability of success always decreases with respect to increasing the acceleration and also with respect to decreasing the presence probability of particle in right Rindler region. The mutual information and the concurrence behave in the same way for small values of acceleration and large values of presence probability of particle in right Rindler region. Nevertheless, when the particle has larger probability of being in the left Rindler region and for the large values of the acceleration, the mutual information and the concurrence have different behaviours as compared to the probability of success with respect to the acceleration and with respect to the presence probability of particle in the right Rindler region. Therefore, the mutual information and the concurrence are not reliable measures for these ranges of the acceleration and the presence probability of particle in right Rindler region. In fact, the concurrence may not be served as a suitable entanglement for superdense coding, at least, for the special ranges of the acceleration and the presence probability of particle in the right Rindler region.

Recently, it has been shown that the relativistic effects entering the description of the dynamics such as frame dependence, time dilation, and Doppler shift, already existent in inertial motion can compete with or even overwhelm the effect due to uniform acceleration in a quantum field [12]. In fact, the relativistic effects (frame dependence, time dilation, and Doppler shift), the environmental influences (quantum decoherence, entanglement dynamics) and another issues, discussed in [12] can be took account into superdense coding in a fully relativistic system, in the interaction region of localized objects and quantum fields. Thus, the results of this paper can be checked for estimation of the efficiency of superdense coding in relativistic quantum systems under environmental influences. These issues would be interesting but lies beyond the scope of the present work.

Also, the transmission of both classical and quantum information between two arbitrary observers has been investigated in globally hyperbolic spacetimes using a quantum field as a communication channel [13]. In this situation, study of the superdense coding is desirable. We will return to the issue raised by this paper with a detailed discussion of such a situation and to address to which the superdense coding can be affected by a globally hyperbolic spacetime.

\section{REFERENCES}

[1] Alsing P. M., Fuentes-Schuller I., Mann R. B., Tessier T. E. "Entanglement of Dirac fields in noninertial frames". Phys. Rev. A. (2006); 74: 032326.

[2] Mehri-Dehnavi H., Mirza B., Mohammadzadeh H., Rahimi R. "Pseudo-entanglement evaluated in noninertial frames". Ann. Phys. (2011); 326: 1320.

[3] Mehri-Dehnavi H., Rahimi R., Mohammadzadeh H., Ebadi Z., Mirza B. "Quantum teleportation with nonclassical correlated states in noninertial frames".Quantum Inf. Process. (2015); 14: 1025.

[4] Bruschi D. E., Louko J., Martín-Martínez E., Dragan A., 
Fuentes I. "The Unruh effect in quantum information beyond the single-mode approximation". Phys. Rev. A. (2010); 82: 042332 .

[5] Farahmand M., Mohammadzadeh H., Mehri-Dehnavi H., Rahimi R. "Superdense coding with uniformly accelerated particle". Int. J. Theor. Phys. (2017); 56: 706.

[6] Farahmand M., Mohammadzadeh H., Rahimi R, Mehri-Dehnavi H. "Residual entanglement of accelerated fermions is useful". Ann. Phys. (2017); 383: 389.

[7] Groisman B., Popescu S., Winter A. "Quantum, classical, and total amount of correlations in a quantum state". Phys. Rev. A. (2005); 72: 032317.

[8] Wootters W. K. "Entanglement of formation of an arbitrary state of two qubits". Phys. Rev. Lett. (1998); 80: 2245.
[9] Bennett C. H., Wiesner S. J. "Communication via one-and two-particle operators on Einstein-Podolsky-Rosen states". Phys. Rev. Lett. (1992); 69: 2881.

[10] Rahimi R., Takeda K., Ozawa M., Kitagawa M. "Entanglement witness derived from NMR superdense coding". J. Phys. A (2006); 39: 2151.

[11] Einstein A., Podolsky B., Rosen N. "Can quantum-mechanical description of physical reality be considered complete?" Phys. Rev. (1935); 47: 777.

[12] Lin S. Y., Chou C. H., Hu B. L. "Quantum teleportation between moving detectors" Phys. Rev. D (2015); 91:084063.

[13] Landulfo A. G. S. "Nonperturbative approach to relativistic quantum communication channels" Phys. Rev. D (2016); 93:104019. 